\title{
Leukemia Stem Cell-Released Microvesicles Promote the Survival and Migration of Myeloid Leukemia Cells and These Effects Can Be Inhibited by MicroRNA34a Overexpression
}

\author{
Yue Wang, 1 Qian Cheng, ${ }^{2}$ Jing Liu, ${ }^{2}$ and Min Dong ${ }^{1}$ \\ ${ }^{1}$ Department of Hematology, The Affiliated Hospital of Guilin Medical College, Guilin, Guangxi 541001, China \\ ${ }^{2}$ Department of Hematology, The Third Xiangya Hospital of Central South University, Changsha 410013, China \\ Correspondence should be addressed to Min Dong; dm1175@sina.com
}

Received 27 January 2016; Accepted 7 March 2016

Academic Editor: Weiwen Long

Copyright (C) 2016 Yue Wang et al. This is an open access article distributed under the Creative Commons Attribution License, which permits unrestricted use, distribution, and reproduction in any medium, provided the original work is properly cited.

Leukemia stem cells (LSCs) play the major role in relapse of acute myeloid leukemia (AML). Recent evidence indicates that microvesicles (MVs) released from cancer stem cells can promote tumor growth and invasion. In this study, we investigated whether LSCs-released MVs (LMVs) can regulate the malignance of AML cells and whether overexpression of tumor suppressive microRNA (miR), miR34a, is able to interrupt this process. LSCs were transfected with miRNA control (miRCtrl) or miR34a mimic for producing LMVs, respectively, defined as LMVs ${ }^{\text {miRCtrl }}$ and LMVs ${ }^{\text {miR34a }}$. The effect of miR34a transfection on LSC proliferation and the effects of LMVs ${ }^{\text {miRCtrl }}$ or LMVs ${ }^{\text {miR34a }}$ on the proliferation, migration, and apoptosis of AML cells (after LSC depletion) were determined. The levels of miR34a targets, caspase-3 and T cell immunoglobulin mucin-3 (Tim-3), were analyzed. Results showed that (1) LMVs ${ }^{\text {miRCtrl }}$ promoted proliferation and migration and inhibited apoptosis of AML cells, which were associated with miR34a deficit; (2) transfection of miR34a mimic inhibited LSC proliferation and increased miR34a level in LMVs ${ }^{\text {miR34a }}$; (3) LMVs ${ }^{\text {miR34a }}$ produced opposite effects as compared with LMVs ${ }^{\text {miRCtrl }}$, which were associated with the changes of caspase-3 and Tim-3 levels. In summary, LMVs support AML cell malignance and modulating miR34a could offer a new approach for the management of AML.

\section{Introduction}

Acute myeloid leukemia (AML) is an aggressive disease characterized by rapid proliferation of immature myeloid cells in the bone marrow resulting in dysfunctional hematopoiesis [1]. Although current treatments can induce remission, many AML patients end in relapse owing to the presence of dormant leukemia stem cells (LSCs) which are resistant to chemotherapies. LSCs are able to self-renew, proliferate, and differentiate extensively, which are crucial for the initiation and maintenance of AML. Cancer stem cells are known to affect cancer progression in part through secretory factors [2]. Microvesicles (MVs) are membrane vesicles that are released from cells undergoing stress, activation, or apoptosis, which can mediate cell-to-cell communication by transferring proteins, mRNAs, and microRNAs (miRs) and lipids between cells $[3,4]$. Recent evidence has shown that MVs secreted from cancer stem cells could interact with surrounding cancer cells and stromal cells, which may be involved in tumor progression and metastasis $[5,6]$. For example, gastric cancer stem cell-secreted MVs were shown to promote gastric cancer cell proliferation and migration [5]. Moreover, MVs secreted from renal cancer stem cells induced in vitro and in vivo angiogenesis and favored lung metastasis [6]. However, it is unknown whether and how LSCs-derived MVs (LMVs) regulate the malignance of AML.

miRs are small noncoding RNAs that function as major players of posttranscriptional gene regulation within diverse cell types. Dysregulation of tumor-suppressive miR34a has been implicated in AML [7]. Forced expression of miR34a resulted in granulocytic differentiation of AML blasts, suggesting that increasing miR34a levels might become therapeutically useful for AML [8]. In addition, several miR34a downstream target genes were identified in AML. For 
example, caspase-3 was reported as an essential molecule for programmed cell death, differentiation, and survival of AML $[9,10]$. T cell immunoglobulin mucin-3 (Tim-3) was highly expressed in human AML cells, which could trigger prosurvival and proinvasive signaling [11, 12]. Since MVs may act as paracrine or endocrine mediators by a horizontal transfer of genetic information into the recipient cells [13], we proposed that overexpression of miR34a in LSCs could synchronously increase the level of miR34a in LMVs, which could be able to abrogate LMVs-induced effects on AML.

In this study, we aimed to investigate the effects of LMVs on the proliferation, migration, and apoptosis of AML cells. The miR34a associated caspase- 3 and Tim-3 pathways in LMVs-mediated effects were also investigated.

\section{Materials and Methods}

2.1. Cell Culture and Sorting. Human AML cell line KGla (ATCC, Manassas, VA) was maintained in suspension culture with Dulbecco's Modified Eagle's medium (DMEM) supplemented with $10 \%$ fetal bovine serum (FBS) and $100 \mathrm{U} / \mathrm{mL}$ of penicillin and streptomycin. Since LSCs were defined as CD34+CD38- fraction of KGla cells and all of KGla cells were CD34+, LSCs were enriched by indirectly labeling with CD38-FITC antibody and anti-FITC microbeads according to the manufacturer's instructions (Miltenyi Biotec, Bergisch Gladbach, Germany). Briefly, cell suspensions were centrifuged at $125 \mathrm{~g}$ for $10 \mathrm{~min}$ and washed in phosphate-buffered saline (PBS). Then, cell pellets were resuspended in separating buffer (PBS containing $0.5 \%$ bovine serum albumin) and incubated with CD38-FITC antibody for $30 \mathrm{~min}$. After washing with PBS, cell pellets were resuspended in separating buffer containing Anti-FITC MicroBeads for $15 \mathrm{~min}$. After several washing steps, filtrates (CD34+CD38- cells) were collected by a LD column using a MidiMACS separator system. Throughout the sorting procedure, cells were kept at $4^{\circ} \mathrm{C}$ and analyzed immediately by flow cytometry. The remaining cells after depletion of LSCs were defined as AML cells, which were used for coculture studies.

2.2. Flow Cytometry Analysis. $1 \times 10^{5}$ cells were resuspended in PBS and incubated with antibodies against LSC surface antigens including CD34 and CD38 for $30 \mathrm{~min}$ at $4^{\circ} \mathrm{C}$. The IgG isotype was served as negative control. LSCs were used to generate LMVs for coculture with AML cells.

2.3. Cell Transfection. $5 \times 10^{5}$ cell suspensions were seeded in 6-well plates and transfected with miR34a mimic (Sigma, $100 \mathrm{nM}$ ) or miR control (miRCtrl; Sigma, $100 \mathrm{nM}$ ) using Lipofectamine 2000 transfection reagent (Invitrogen, CA) for 2 days. Transfection efficacy was examined by Real-Time Quantitative Reverse Transcription PCR (qRT-PCR).

2.4. $q R T-P C R$. Total RNA was extracted with TRIzol Reagent (Invitrogen) according to the manufacturer's instructions. cDNA was synthesized using miScript reverse transcription kit (Qiagen). Quantitative real-time PCR was conducted with miR34a specific primers and miScript SYBR Green
PCR Kit (QIAGEN) on a real-time PCR system (Bio-Rad). Small nuclear RNA U6 was used as an internal control. All experiments were carried out in triplicate. Relative expression of miR34 was calculated using the $2^{-\Delta \Delta \mathrm{CT}}$ method [14].

2.5. Isolation and Identification of LMVs ${ }^{\text {miRCtrl }}$ and $L M V s^{m i R 34 a}$. MVs generated from LSCs transfected with miRCtrl or miR34a mimic were, respectively, defined as LMVs ${ }^{\text {miRCtrl }}$ and LMVs ${ }^{\text {miR34a }}$. MVs were collected from cell culture medium and purified by differential centrifugations as previously reported [15]. The particle number and size distribution of secreted MVs were measured by NanoSight NS300 instrument (Malvern Instruments, Amesbury, UK) according to the manufacturer's instructions [16].

2.6. Coculture of AML Cells with Vehicle, LMVs ${ }^{\text {miRCtrl }}$, or $L M V s^{m i R 34 a}$. Isolated LMVs ${ }^{\text {miRCtrl }}$ or LMVs ${ }^{\text {miR34a }}(50 \mu \mathrm{g})$ were resuspended with fresh culture medium. AML cells were, respectively, treated with vehicle (veh, fresh culture medium), LMVs ${ }^{\text {miRCtrl }}$, and LMVs ${ }^{\text {miR34a }}$ for $24 \mathrm{hrs}$ in an incubator $\left(37^{\circ} \mathrm{C}, 5 \% \mathrm{CO}_{2}\right)$. AML cells were collected and subjected to cell apoptosis, proliferation, and migration assays.

2.7. Cell Apoptosis Assay. The terminal deoxynucleotidyl transferase-mediated dUTP-digoxigenin nick end labeling (TUNEL) assay was performed according to the manufacturer's instructions (Boehringer Mannheim, Germany). In brief, cells were fixed with $4 \%$ paraformaldehyde for $30 \mathrm{~min}$ and subsequently permeabilized with $0.1 \%$ Triton X-100 in $0.1 \%$ sodium citrate for $2 \mathrm{~min}$. The cells were incubated with $50 \mu \mathrm{L}$ TUNEL reaction mixture for $1 \mathrm{~h}$ at $37^{\circ} \mathrm{C}$. Labeled cells were analyzed by flow cytometry.

2.8. Cell Proliferation Assay. Cells were seeded in 96-well plate and added with $10 \mu \mathrm{L}$ methyl thiazolyl tetrazolium (MTT, 12 mM; Invitrogen, NY) solution. After 4 hrs of incubation, dimethyl sulfoxide- (DMSO-) isopropanol (1:1) solvent was added directly to the cell suspension and incubated for $30 \mathrm{~min}$. The absorbance was measured at $570 \mathrm{~nm}$.

2.9. Cell Migration Assay. Cells were suspended in serumfree medium and added to the top chambers of 24-well transwell plates (Corning Inc., NY). The lower chambers were filled with $500 \mu \mathrm{L}$ complete culture media. Transwell plates were incubated for $24 \mathrm{hrs}$ in a $37^{\circ} \mathrm{C}$ cell incubator. Migrated cells were fixed with $4 \%$ paraformaldehyde, stained with $0.1 \%$ crystal violet, and enumerated in ten randomly selected fields under a light microscope.

2.10. Western Blot. Proteins were extracted from cells with lysis buffer containing protease inhibitor (Roche Diagnostics). Proteins were separated by SDS-PAGE and transferred onto PVDF membrane. The PVDF membrane was blocked with $5 \%$ nonfat milk in $1 \mathrm{x}$ Tris-buffered saline with Tween20 (TBST, pH 7.6) at room temperature (RT) for $1 \mathrm{~h}$ and then incubated with primary antibody against Tim-3 (1:200; Santa Cruz) at $4^{\circ} \mathrm{C}$ overnight. $\beta$-actin (1:40000; Sigma) was 
used to normalize protein loading. After being washed with TBST thrice, membranes were incubated with horseradish peroxidase- (HRP-) conjugated IgG (1:40000, Jackson Lab) for 1 hour at RT. Blots were then developed with enhanced chemiluminescence developing solutions and quantified.

2.11. Enzyme-Linked Immunosorbent Assay (ELISA). Caspase-3 activity in AML cells from different groups was assayed using Quantikine caspase-3 (R\&D System) as previously reported [17]. In brief, AML cells were harvested, washed in PBS, and resuspended in extraction buffer containing protease inhibitors. Standard and sample extracts were added to the microplate precoated with antibody specific for caspase-3. HRP substrate was added to each well. The level of caspase- 3 was measured at $450 \mathrm{~nm}$.

2.12. Statistical Analysis. Statistical analysis was done using SPSS software version 16.0. All experiments were carried out at least three times. Data are shown as mean \pm SEM, unless otherwise noted. Comparison for two groups was examined by Student's $t$-test. Multiple comparisons were performed by one-way ANOVA. For all cases, statistical significance was set as $P<0.05$.

\section{Results}

3.1. Characterization of LSCs. To determine the proportion of LSCs in KGla cells and whether LSCs were successfully isolated from KGla cells, KGla cells and microbeadsisolated LSCs were stained with CD34-PE and CD38-FITC antibodies $[18,19]$ and analyzed by flow cytometry. As shown in Figure 1(a), the KGla cells contained about 32.8\% of CD34+CD38- cells. After microbead sorting procedure, more than $95 \%$ of the cells were CD34+CD38- and considered LSCs.

3.2. The Effect of miR34a Overexpression on LSC Proliferation. The transfection efficiency of miR34a mimic was evaluated by qRT-PCR (Figure 1(b)). As expected, the level of miR34a in LSCs was significantly elevated after miR34a mimic transfection when compared with miRCtrl transfection or veh. This finding indicates successful overexpression of miR34a in LSCs. Overexpression of miR34a remarkably inhibited the proliferation ability of LSCs (versus control, $P<0.05$; versus miRCtrl, $P<0.05$; Figure 1(c)).

3.3. The Particle Concentration and miR34a Levels of LMVs, $L M V^{m i R C t r l}$, and $L M V s^{m i R 34 a}$. As shown in Figure 2(a), NTA analysis showed that the number of LMVs ${ }^{\mathrm{miR} 34}$ was higher than that of LMVs or LMVs ${ }^{\text {miRCtrl }}$, although there were no significant changes in their size distribution. More interestingly, the expression of miR34a in LMVs was displayed in the same pattern as their parent LSCs. As shown in Figure 2(a), qRT-PCR assay showed that the level of miR34a in LMVs ${ }^{\text {miR34 }}$ was much higher than that of LMVs or $\mathrm{LMVs}^{\mathrm{miRC} \text { trl }}$. There was no significant difference of miR34a level between LMVs and LMVs ${ }^{\text {miRCtrl }}$.
3.4. The Effects of LMVs ${ }^{\text {miRCtrl }}$ and LMVs ${ }^{\text {miR34a }}$ on Apoptosis, Proliferation, and Migration of AML Cells. AML cell apoptosis (Figure 3 ) was identified by TUNEL staining and analyzed by flow cytometry. The apoptosis in AML cells was decreased after coincubation with LMVs ${ }^{\text {miRCtrl }}$ when compared with veh or LMVs ${ }^{\text {miR34a }}(11.29 \pm 1.59 \%$ versus $26.70 \pm 1.90 \%, P<$ 0.05 versus veh; $11.29 \pm 1.59 \%$ versus $24.53 \pm 2.30 \%, P<0.05$ versus $\mathrm{LMVs}^{\mathrm{miR} 34 \mathrm{a}}$; and $n=3$ ). There was no significant difference in apoptosis between veh-treated and LMVs ${ }^{\text {miR34a }}$ treated AML cells $(26.70 \pm 1.90 \%$ versus $24.53 \pm 2.30 \%, P=$ $0.63 ; n=3$ ). Moreover, the proliferation (Figure 4(a)) and migration abilities (Figure 4(b)) of AML cells were enhanced after coincubation with LMVs ${ }^{\text {miRCtrl }}(P<0.05$ versus veh; $n=$ 3 ) whereas these effects were absent in AML cells coincubated with LMVs $^{\text {miR34a }}(P>0.05$ versus veh; $n=3)$.

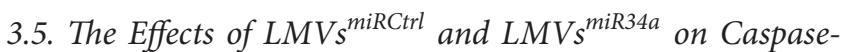
3 Activity and Tim-3 Expression in AML Cells. The activity of caspase-3 was reduced in AML cells coincubated with LMVs $^{\text {miRCtrl }}\left(P<0.05\right.$ versus veh; Figure 5(a)). LMVs ${ }^{\text {miR34a }}$ significantly increased the activity of caspase-3 in AML cells $(P<0.05$ versus veh; Figure 5(a)). The expression of Tim-3 was upregulated in LMVs ${ }^{\text {miRCtrl }}$-treated AML cells $(P<0.05$ versus veh; Figure $5(\mathrm{~b}))$ but was downregulated in LMVs ${ }^{\text {miR34a }}$-treated AML cells $(P<0.05$ versus veh; Figure 5(b)).

\section{Discussion}

There are several major findings in this study. Firstly, we demonstrated that LMVs promote the proliferation, migration, and survival of AML cells, which is associated with miR34a deficit since overexpression of miR34a in LSCs inhibited their proliferation. Secondly, LSCs with miR34a mimic transfection generate LMVs containing high level of miR34a (e.g., LMVs ${ }^{\text {miR34a }}$ ), which exert opposite effects on AML cells as compared to LMVs. Thirdly, the different effects of LMVs and LMVs ${ }^{\text {miR34a }}$ on AML cells were associated with their ability to differently regulate miR34a-targeted caspase-3 and Tim-3 pathways.

The concept of cancer stem cells has been proposed for many years and received increasing attention in recent years. There is solid evidence that AML contains a subpopulation of LSCs that are largely responsible for the refractory and resistance of AML to chemotherapy and immunotherapy [18, $20,21]$. However, the underlying mechanisms have not been fully understood. In our study, we isolated the CD34+CD38fraction (LSCs) from KGla cells by using the immunemagnetic microbead method as previously reported [18] and cultured for generating MVs. Elevated level of MVs has been found in various diseases including cancer, suggesting that they may serve as diagnostic and prognostic tool [22]. In addition, MVs can act as autocrine or paracrine mediators since they can merge with target cells to exert a wide range of actions [13] whereas whether LMVs have effects on the survival and malignance of AML remains unknown. In our study, we discovered for the first time that LMVs can 

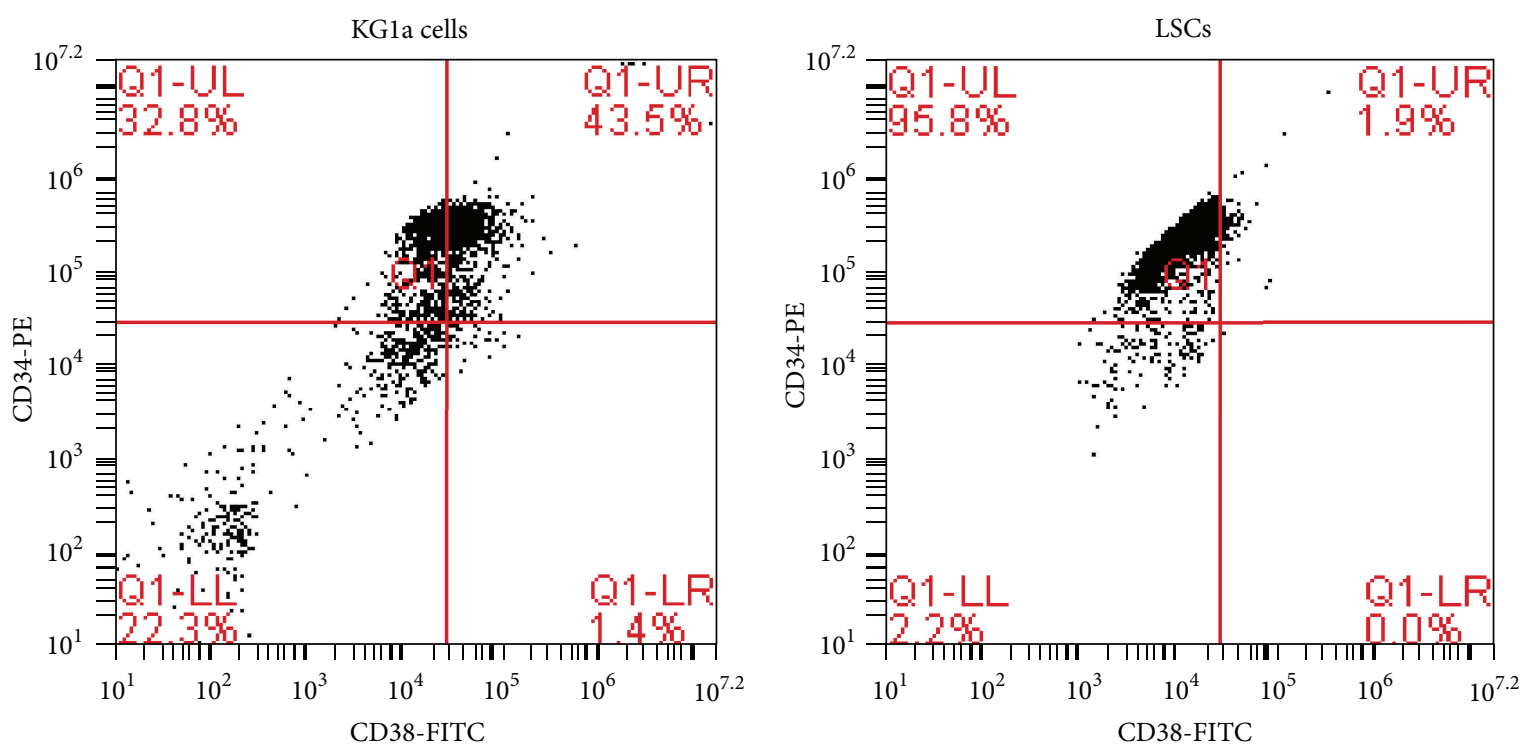

(a)

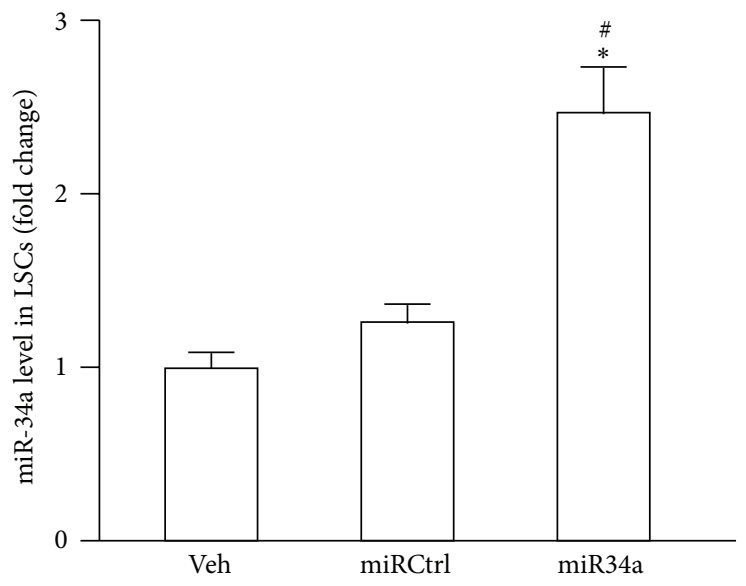

(b)

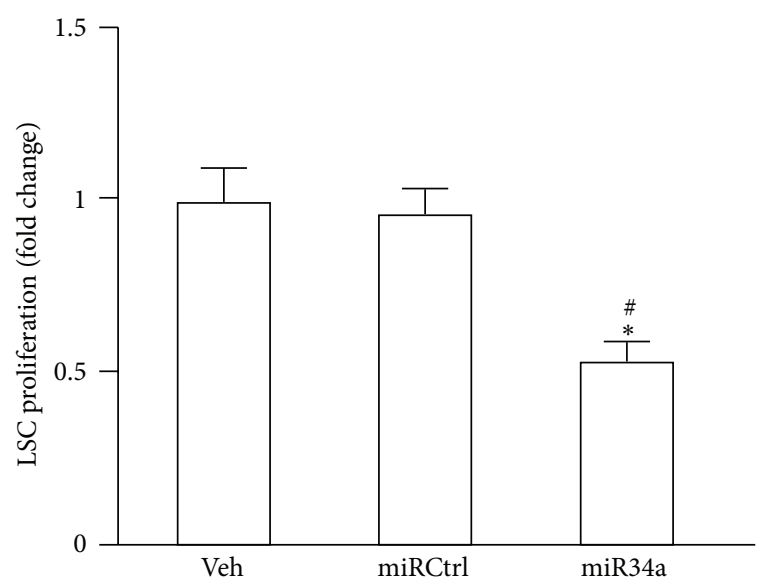

(c)

FIGURE 1: LSC characterization and the effect of miR34a overexpression on LSC proliferation. (a) Representative flow cytometric plots of CD34-PE versus CD38-FITC. Expressions of CD34 and CD38 in KGla cells before sorting (left) and after sorting (right). (b) LSCs were subjected to vehicle (veh) treatment and transfected with miRCtrl or miR34a mimic as indicated and 2 days later the miR34a level was measured by qRT-PCR. (c) LSCs were subjected to treatment with veh or miRCtrl transfection or miR34a mimic transfection. After 2 days, the LSC proliferation was determined by MTT assay. Data represents mean \pm SEM. ${ }^{*} P<0.05$ versus veh; ${ }^{\#} P<0.05$ versus miRCtrl.

promote the proliferative and migrative abilities and prevent the apoptosis of AML cells. A recent study has demonstrated that MVs derived from renal cancer stem cells induced abnormal endothelial cell growth, invasion of matrix, and resistance to apoptosis [6]. Therefore, our findings provide novel insight into previous perspectives [5, 6], suggesting that MVs derived from cancer stem cells can change the phenotypes of surrounding tumor and stromal cells to create a favorable tumor microenvironment.

Next, we examined the potential mechanisms of LMVsinduced effects on AML cells. It has been recently proposed that cancer stem cells not only initiate cancers but also support the progression of cancer in virtue of their oncogenic contents [23]. MVs are considered ideal vehicles for transferring the contents of cancer stem cells to bystander cells. Previous studies have also suggested that the RNA contents (mainly miRs and mRNAs) of MVs play a critical role, since the RNase treatment of MVs significantly inhibited the biological effects of MVs [6]. We therefore hypothesize that reprogramming the miR contents of LMVs could be able to alter the effects of LMVs on AML cells. Tumor-suppressive miR34a has been reported to be downregulated in most cancers, and restoration of miR34a has been shown to induce apoptosis and enhance chemosensitivity of cancer cells [24, 25]. Our current study found that overexpression of miR34a in LSCs not only significantly inhibited their proliferative ability but also produced LMVs containing high level of miR34a which could inhibit the effects of LMVs on AML cells. These data suggest a potential role of miR34a in the maintenance of LSCs and in LMVs-mediated effects, which may offer a novel way to target LSCs as well as modulate the interaction between LSCs and AML cells. 

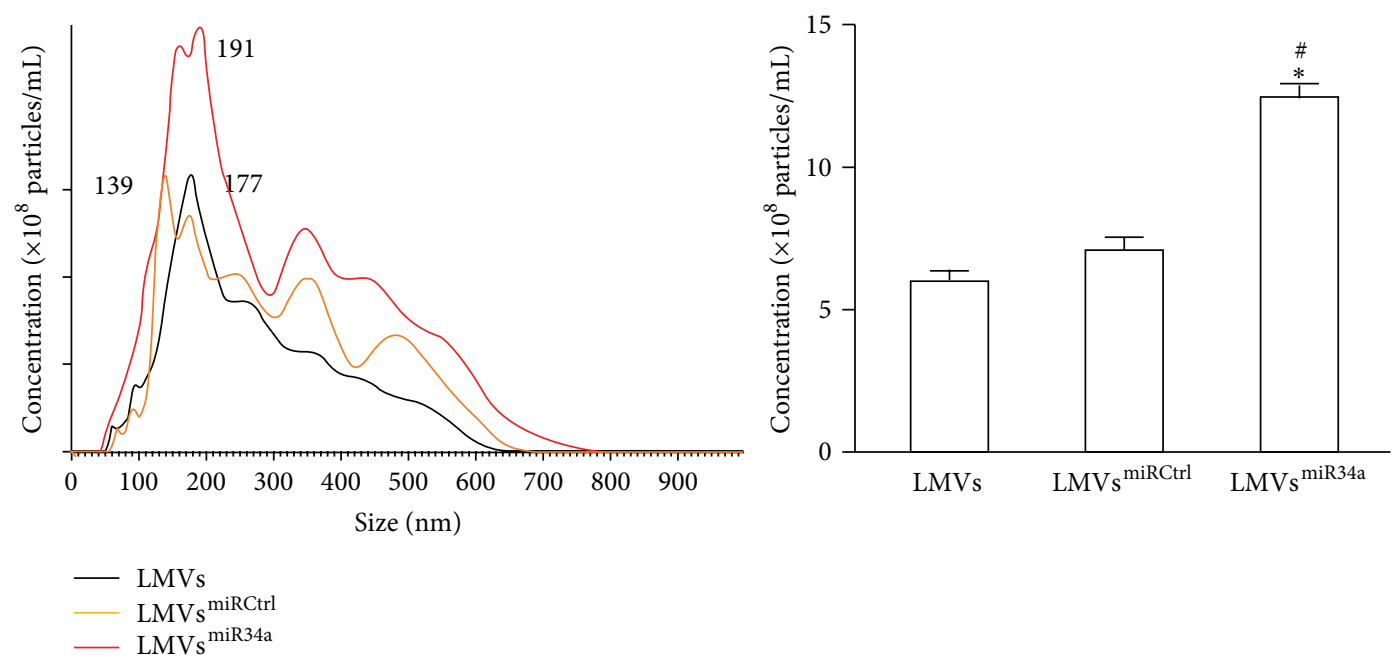

(a)

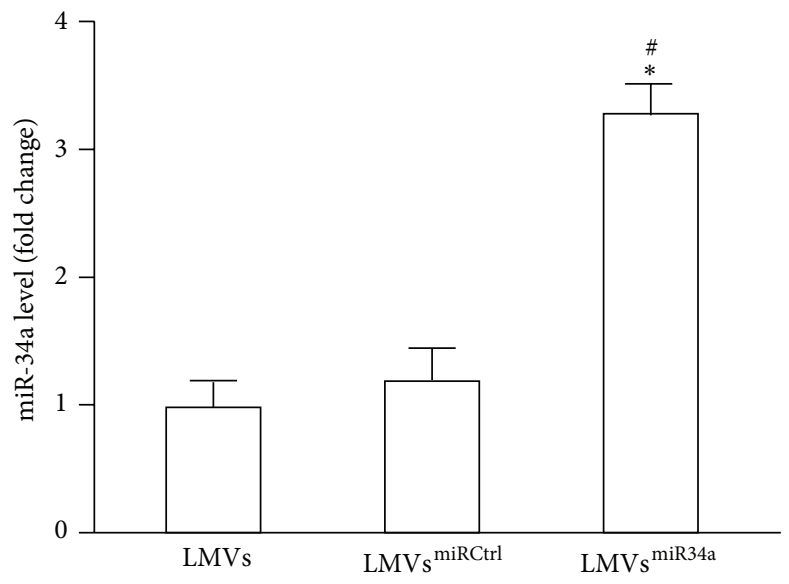

(b)

FIgURE 2: The particle number and miR-34a level of LMVs, LMVs ${ }^{\text {miRCtrl }}$, and LMVs ${ }^{\text {miR34a }}$. (a) Representative NTA plots of MV size distribution (left) and concentration (right). (b) qRT-PCR analysis of miR-34a level in LMVs, LMVs ${ }^{\text {miRCtrl }}$, and LMVs ${ }^{\text {miR34a }}$. Data represents mean \pm SEM. ${ }^{*} P<0.05$ versus LMVs; ${ }^{\#} P<0.05$ versus LMVs ${ }^{\text {miRCtrl }}$. LMVs represent MVs generated from LSCs without transfection; LMVs ${ }^{\text {miRCtrl }}$ represent MVs generated from LSCs transfected with miRCtrl; and LMVs ${ }^{\text {miR34a }}$ represent MVs generated from LSCs transfected with miR34a mimic.

The downstream target genes of miR34a such as caspase3 and Tim-3 have been identified in AML [9-12]. As the key effector of cellular death, caspase-3 activity is a predictor of survival in AML $[9,10]$. A previous study has reported that overexpression of miR34a significantly induced pancreatic $\beta$ cell apoptosis accompanied with increased caspase-3 activity [26]. In our study, we found that LMVs could significantly decrease caspase-3 activity in AML cells, which was inhibited by miR34a overexpression. Tim-3, highly expressed in human AML cells, can be served as a promising candidate for AML therapy $[11,12]$. A previous report has shown that the high expression of Tim-3 is correlated with higher metastatic potential and shorter overall survival of cervical cancer [27]. Search for miR targets by using TargetScan confirmed that Tim-3 has a putative miR34a binding site within its $3^{\prime}$ UTR. We found that LMVs significantly upregulated Tim-3 protein expression in AML cells and these effects were reversed by LMVs carrying high level of miR34a. However, we did not directly test whether miR34a can target Tim-3 mRNA by binding to its $3^{\prime}$ UTR and investigated whether overexpression of Tim-3 protein could rescue the effects of miR34a overexpression at this time. We acknowledge that there are some limitations in our study and it would be necessary to incorporate these questions into our future work. Of particular note, miR34a overexpression could partially block the effects of LMVs on AML cell proliferation, migration, and proliferation, although it was able to significantly elevate caspase- 3 activity and decrease Tim- 3 expression in AML cells; these data suggests that other miRs and associated mechanisms may also participate in LMVs-induced effects. Altogether, the involvements of miR34a in LMVs and its downstream targets, caspase- 3 and Tim-3, in the malignance 

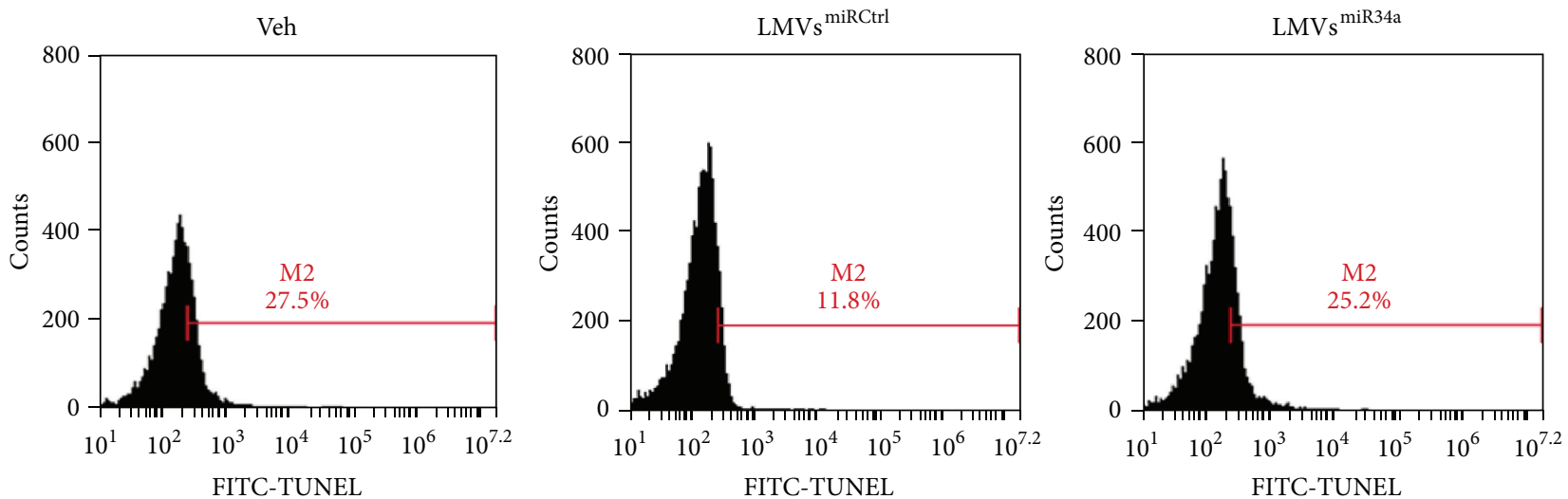

(a)

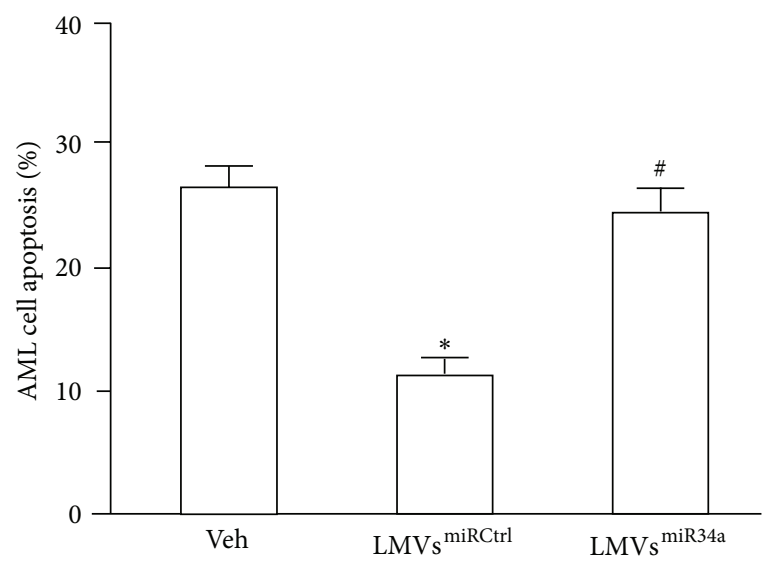

(b)

FIGURE 3: Effects of LMVs ${ }^{\text {miRCtrl }}$ and LMVs ${ }^{\text {miR34a }}$ on AML cell apoptosis. (a) Representative flow cytometric plots of AML cell apoptosis (percentage of FITC-TUNEL positive cells) after coincubation with veh, LMVs ${ }^{\text {miRCtrl }}$, or LMVs ${ }^{\text {miR34a }}$. (b) Summarized data of AML cell apoptosis. Data represents mean \pm SEM. ${ }^{*} P<0.05$ versus veh; ${ }^{*} P<0.05$ versus LMVs ${ }^{\text {miRCtrl }}$. Veh: fresh culture medium; LMVs ${ }^{\text {miRCtrl }}:$ LMVs generated from miRCtrl transfected LSCs; and LMVs ${ }^{\text {miR34a }}$ : LMVs generated from miR34a mimic transfected LSCs.

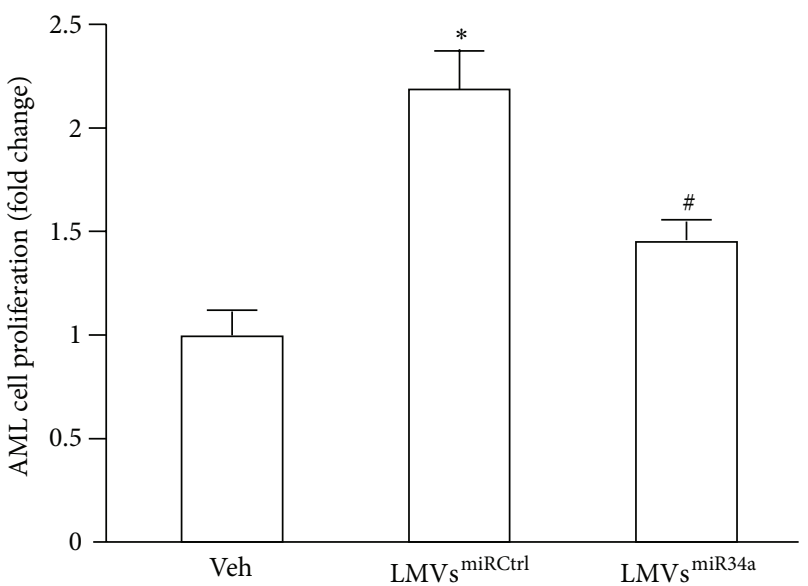

(a)

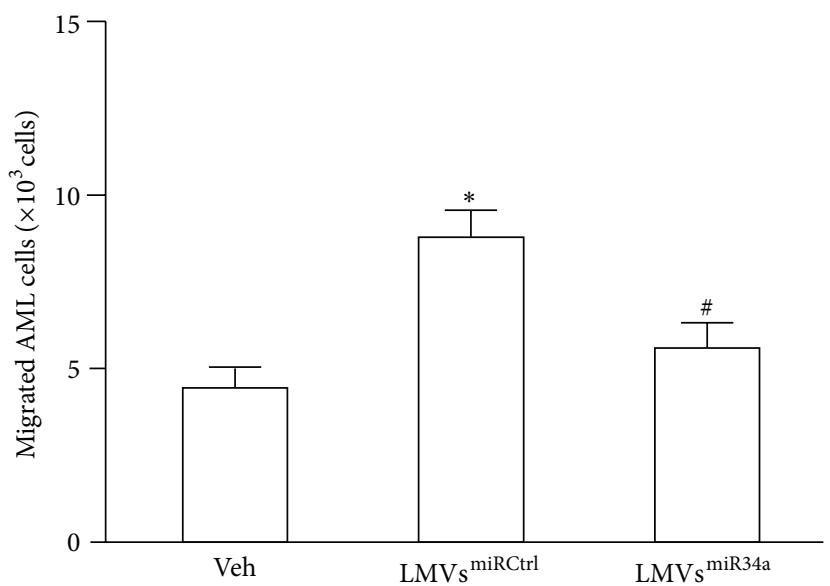

(b)

FIGURE 4: Effects of LMVs ${ }^{\text {miRCtrl }}$ and LMVs ${ }^{\text {miR34a }}$ on AML cell proliferation and migration. (a) Summarized data of AML cell proliferation which was determined by MTT assay. (b) Summarized data of AML cell migration which was determined by transwell assay. Data represents mean \pm SEM. ${ }^{*} P<0.05$ versus veh; ${ }^{\#} P<0.05$ versus LMVs ${ }^{\text {miRCtrl }}$. Veh: fresh culture medium; LMVs ${ }^{\text {miRCtrl }}$ : LMVs generated from miRCtrl transfected LSCs; and LMVs ${ }^{\text {miR34a }}$ : LMVs generated from miR34a mimic transfected LSCs. 


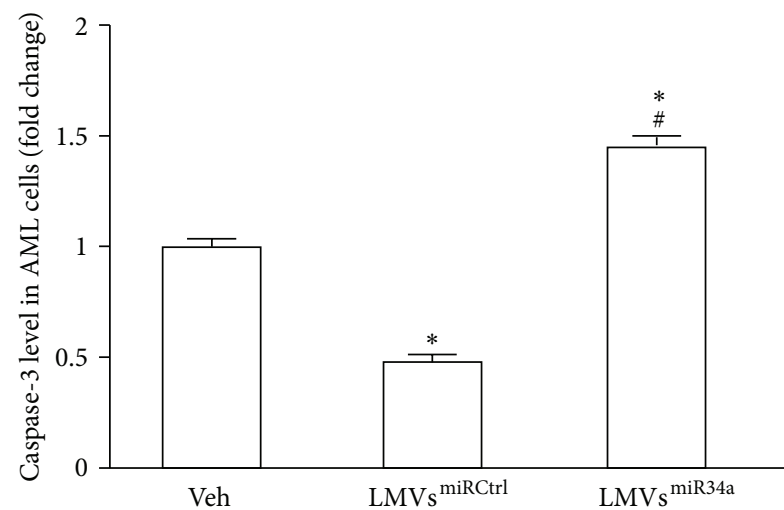

(a)

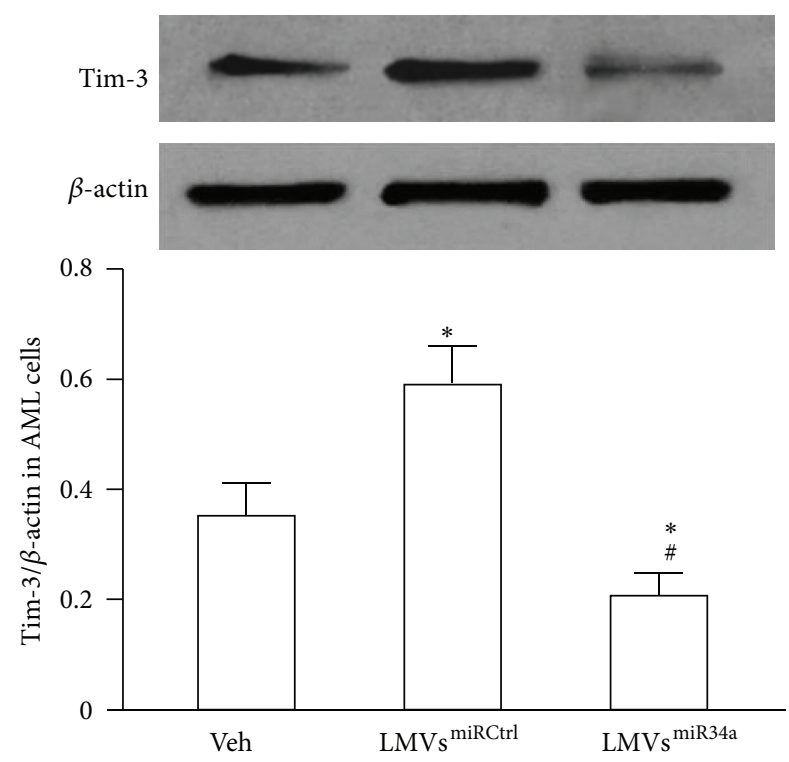

(b)

FIGURE 5: Effects of LMVs ${ }^{\text {miRCtrl }}$ and LMVs ${ }^{\text {miR34a }}$ on the levels of caspase- 3 and Tim-3 protein in AML cells. (a) The activity of caspase-3 in AML cells which was determined by ELISA. (b) The expression of Tim-3 protein in AML cells which was determined by western blot. Data represents mean \pm SEM. ${ }^{*} P<0.05$ versus veh; ${ }^{\#} P<0.05$ versus LMVs ${ }^{\text {miRCtrl }}$. Veh: fresh culture medium; LMVs ${ }^{\text {miRCtrl }}$ : LMVs generated from miRCtrl transfected LSCs; and LMVs ${ }^{\text {miR34a }}$ : LMVs generated from miR34a mimic transfected LSCs.

of AML were demonstrated in our study. Nevertheless, the detailed mechanisms of the roles of LMVs may need further investigation.

\section{Conclusion}

Our data demonstrate that LMVs are able to promote the proliferation and migration and inhibited apoptosis of AML cells, which is associated with the deficit of miR34a. Restoration of miR34a not only inhibits the LSC proliferation but also inhibits these effects of LMVs on AML cells via modulating caspase- 3 and Tim-3 levels. These findings indicate that LMVs support the malignancy of AML cells and targeting of miR34a in LMVs could offer a novel approach for treatment of AML.

\section{Competing Interests}

The authors declare that none of them has any kind of conflict of interests related to the present work.

\section{Acknowledgments}

This work was supported by the National Natural Science Foundation of China (NSFC, no. 81560032 to Min Dong).

\section{References}

[1] C. J. Eaves and R. K. Humphries, "Acute myeloid leukemia and the Wnt pathway," The New England Journal of Medicine, vol. 362 , no. 24, pp. 2326-2327, 2010.
[2] M. A. Velasco-Velázquez, V. M. Popov, M. P. Lisanti, and R. G. Pestell, "The role of breast cancer stem cells in metastasis and therapeutic implications," The American Journal of Pathology, vol. 179, no. 1, pp. 2-11, 2011.

[3] S. F. Mause and C. Weber, "Microparticles: protagonists of a novel communication network for intercellular information exchange," Circulation Research, vol. 107, no. 9, pp. 1047-1057, 2010.

[4] J. Ratajczak, M. Wysoczynski, F. Hayek, A. JanowskaWieczorek, and M. Z. Ratajczak, "Membrane-derived microvesicles: important and underappreciated mediators of cell-to-cell communication," Leukemia, vol. 20, no. 9, pp. 1487-1495, 2006.

[5] M. Wang, C. Zhao, H. Shi et al., "Deregulated microRNAs in gastric cancer tissue-derived mesenchymal stem cells: novel biomarkers and a mechanism for gastric cancer," British Journal of Cancer, vol. 110, no. 5, pp. 1199-1210, 2014.

[6] C. Grange, M. Tapparo, F. Collino et al., "Microvesicles released from human renal cancer stem cells stimulate angiogenesis and formation of lung premetastatic niche," Cancer Research, vol. 71, no. 15, pp. 5346-5356, 2011.

[7] X. Wang, J. Li, K. Dong et al., “Tumor suppressor miR-34a targets PD-L1 and functions as a potential immunotherapeutic target in acute myeloid leukemia," Cellular Signalling, vol. 27, no. 3, pp. 443-452, 2015.

[8] J. A. Pulikkan, P. S. Peramangalam, V. Dengler et al., " $\mathrm{C} / \mathrm{EBP} \alpha$ regulated microRNA-34a targets E2F3 during granulopoiesis and is down-regulated in AML with CEBPA mutations," Blood, vol. 116, no. 25, pp. 5638-5649, 2010.

[9] Y. Lu and G.-Q. Chen, "Effector caspases and leukemia," International Journal of Cell Biology, vol. 2011, Article ID 738301, 8 pages, 2011. 
[10] Z. Estrov, P. F. Thall, M. Talpaz et al., "Caspase 2 and caspase 3 protein levels as predictors of survival in acute myelogenous leukemia," Blood, vol. 92, no. 9, pp. 3090-3097, 1998.

[11] I. G. Silva, B. F. Gibbs, M. Bardelli, L. Varani, and V. V. Sumbayev, "Differential expression and biochemical activity of the immune receptor Tim-3 in healthy and malignant human myeloid cells," Oncotarget, vol. 6, no. 32, pp. 33823-33833, 2015.

[12] A. Prokhorov, B. F. Gibbs, M. Bardelli et al., “The immune receptor Tim-3 mediates activation of PI3 kinase/mTOR and HIF1 pathways in human myeloid leukaemia cells," International Journal of Biochemistry and Cell Biology, vol. 59, pp. 11-20, 2015.

[13] L. Biancone, S. Bruno, M. C. Deregibus, C. Tetta, and G. Camussi, "Therapeutic potential of mesenchymal stem cellderived microvesicles," Nephrology Dialysis Transplantation, vol. 27, no. 8, pp. 3037-3042, 2012.

[14] H. S. Cheng, N. Sivachandran, A. Lau et al., "MicroRNA-146 represses endothelial activation by inhibiting pro-inflammatory pathways," EMBO Molecular Medicine, vol. 5, no. 7, pp. 949-966, 2013.

[15] J. Skog, T. Würdinger, S. van Rijn et al., "Glioblastoma microvesicles transport RNA and proteins that promote tumour growth and provide diagnostic biomarkers," Nature Cell Biology, vol. 10, no. 12, pp. 1470-1476, 2008.

[16] R. A. Dragovic, C. Gardiner, A. S. Brooks et al., "Sizing and phenotyping of cellular vesicles using Nanoparticle Tracking Analysis," Nanomedicine: Nanotechnology, Biology, and Medicine, vol. 7, no. 6, pp. 780-788, 2011.

[17] B. Liagre, J. Bertrand, D. Y. Leger, and J.-L. Beneytout, "Diosgenin, a plant steroid, induces apoptosis in COX-2 deficient K562 cells with activation of the p38 MAP kinase signalling and inhibition of NF-kappaB binding," International Journal of Molecular Medicine, vol. 16, no. 6, pp. 1095-1101, 2005.

[18] M. She, X. Niu, X. Chen et al., "Resistance of leukemic stemlike cells in AML cell line KGla to natural killer cell-mediated cytotoxicity," Cancer Letters, vol. 318, no. 2, pp. 173-179, 2012.

[19] Y. Zhang, S. Y. Zhou, H. Z. Yan et al., "miR-203 inhibits proliferation and self-renewal of leukemia stem cells by targeting survivin and Bmi-1," Scientific Reports, vol. 6, Article ID 19995, 2016.

[20] J. M. Gerber, B. D. Smith, B. Ngwang et al., "A clinically relevant population of leukemic $\mathrm{CD} 34^{+} \mathrm{CD} 38^{-}$cells in acute myeloid leukemia," Blood, vol. 119, no. 15, pp. 3571-3577, 2012.

[21] S. J. Horton and B. J. P. Huntly, "Recent advances in acute myeloid leukemia stem cell biology," Haematologica, vol. 97, no. 7, pp. 966-974, 2012.

[22] H. Julich, A. Willms, V. Lukacs-Kornek, and M. Kornek, "Extracellular vesicle profiling and their use as potential disease specific biomarker," Frontiers in Immunology, vol. 5, article 413, 2014.

[23] X. Zhang, W. Zhu, J. Zhang et al., "MicroRNA-650 targets ING4 to promote gastric cancer tumorigenicity," Biochemical and Biophysical Research Communications, vol. 395, no. 2, pp. 275-280, 2010.

[24] L. Kang, J. Mao, Y. Tao et al., "MicroRNA-34a suppresses the breast cancer stem cell-like characteristics by downregulating Notchl pathway," Cancer Science, vol. 106, no. 6, pp. 700-708, 2015.

[25] W. Duan, Y. Xu, Y. Dong, L. Cao, J. Tong, and X. Zhou, "Ectopic expression of miR-34a enhances radiosensitivity of non-small cell lung cancer cells, partly by suppressing the LyGDI signaling pathway," Journal of Radiation Research, vol. 54, no. 4, pp. 611619, 2013.
[26] X. Lin, H. Guan, Z. Huang et al., "Downregulation of Bcl-2 expression by mir-34a mediates palmitate-induced min 6 cells apoptosis," Journal of Diabetes Research, vol. 2014, Article ID 258695, 7 pages, 2014.

[27] Y. Cao, X. Zhou, X. Huang et al., “Tim-3 expression in cervical cancer promotes tumor metastasis," PLoS ONE, vol. 8, no. 1, Article ID e53834, 2013. 

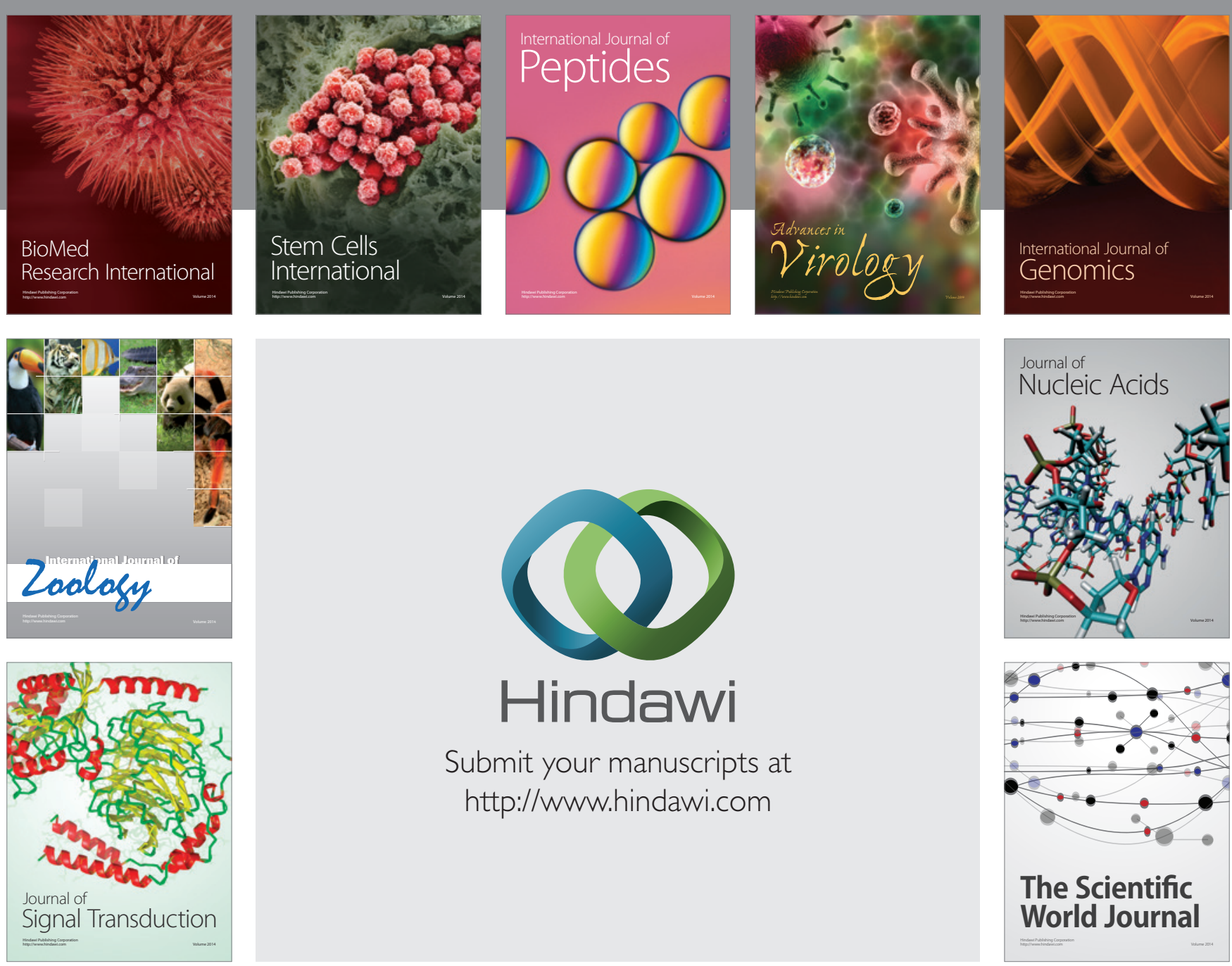

Submit your manuscripts at

http://www.hindawi.com
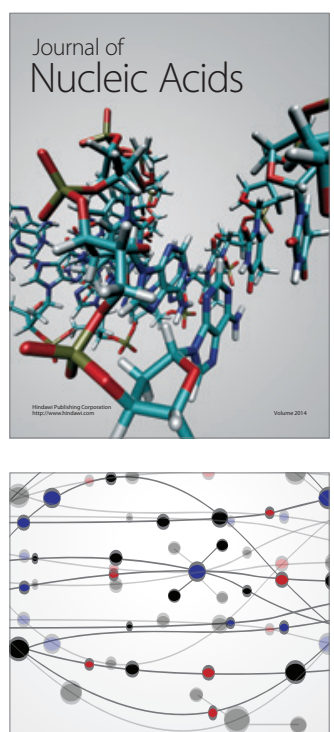

The Scientific World Journal
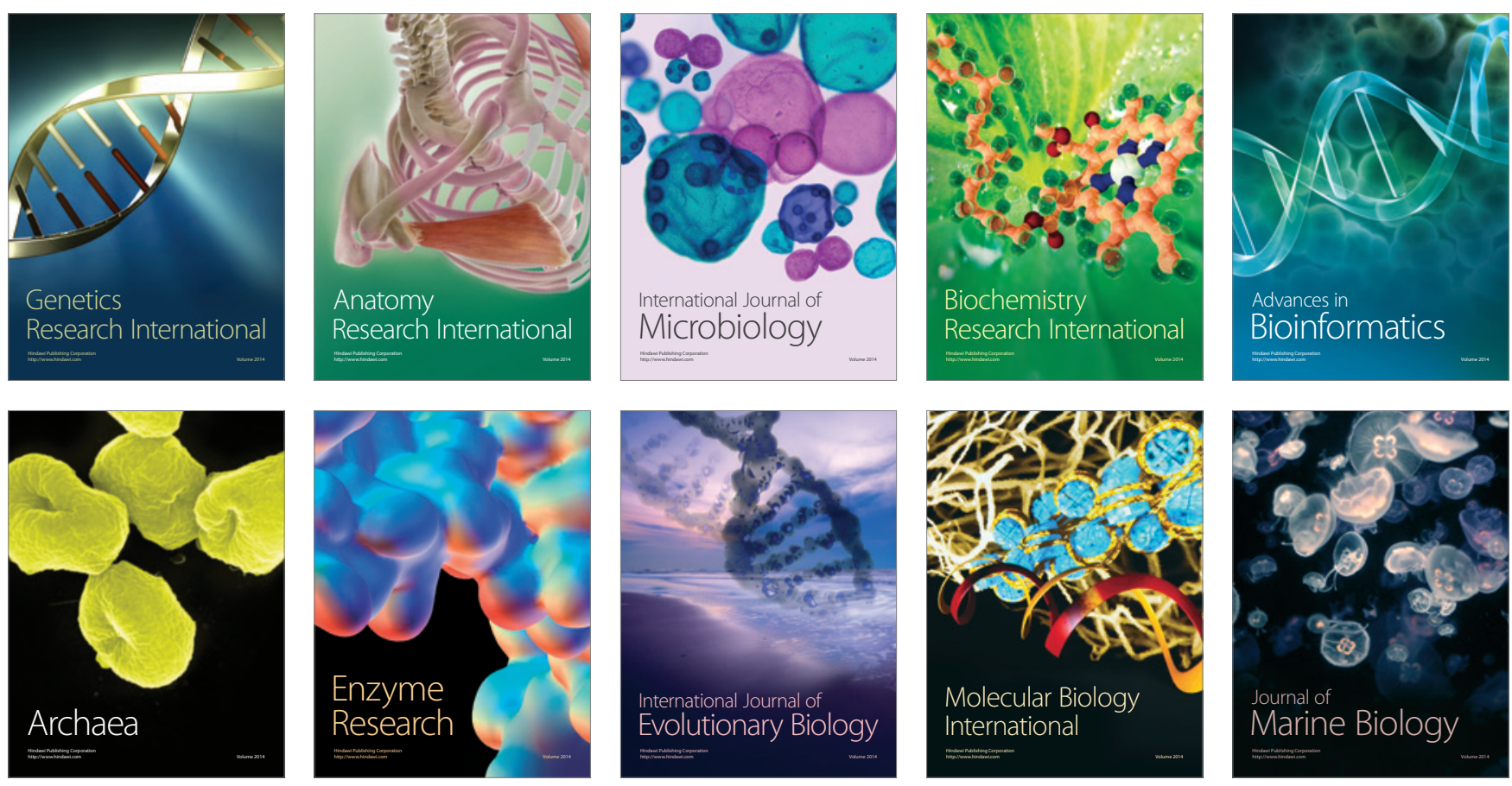